(A) Check for updates

SOLITAIRETM $24 \times 40$
REVASCULARIZATION

DEVICE

COMPLETE STROKE

ZONE COVERAGE ${ }^{1}$

OPHTHALMIC

ALIGNMENT

DISTAL M2

DEPLOYMENT

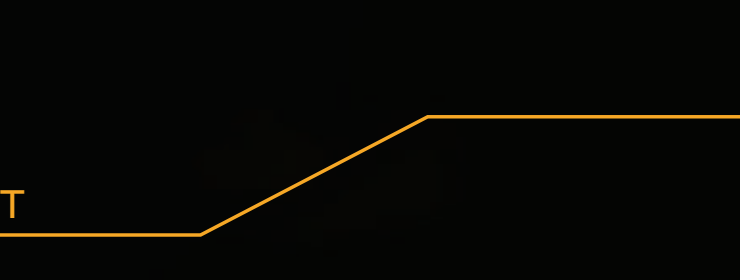

$\square$

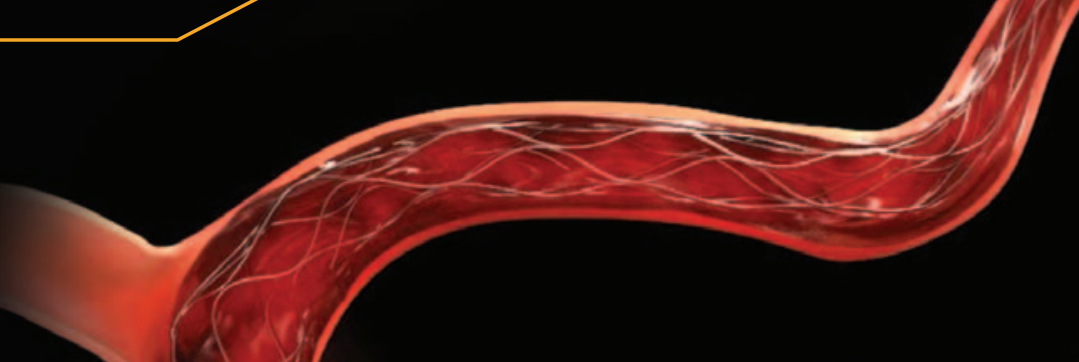

Simplified placement with proximal ophthalmic alignment providing the most complete STROKE ZONE coverage from ICA to M2. ${ }^{1}$

1 Solitaire ${ }^{\mathrm{TM}} 2$ IFU, Trevo ${ }^{\mathrm{TM}}$ Device IFU. Pai S.B., et, al. Microsurgical Anatomy of the Middle Cerebral Artery. Neurology India. June 2005. Vol 53. Issue 2. Keshelava G. et, al. Surgical

anatomy of petrous part of the internal carotid artery. Neuroanatomy (2009) 8: 46-48

(c) 2015 Medtronic. All Rights Reserved. EU-15-525679 - 12/2015

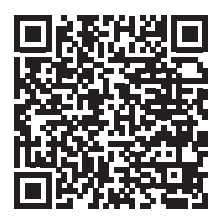

\section{Medtronic}

Further,Together 


\title{
Flow-diverting stents allow efficient treatment of unruptured, intradural dissecting aneurysms of the vertebral artery: An explanatory approach using in vivo flow analysis
}

\author{
Philipp Gölitz ${ }^{1}$, Tobias Struffert ${ }^{1}$, Philip Hoelter ${ }^{1}$, Ilker Eyüpoglu ${ }^{2}$, \\ Frauke Knossalla ${ }^{3}$ and Arnd Doerfler ${ }^{1}$
}

\begin{abstract}
Object: Our study aimed to evaluate the efficiency of flow-diverting stents (FDS) in treating unruptured, intradural dissecting aneurysms of the vertebral artery (VADAs). Additionally, the effect of FDS on the aneurysmal flow pattern was investigated by performing in vivo flow analysis using parametric color coding (PCC).

Methods: We evaluated 11 patients with unruptured, intradural VADAs, treated with FDS. Pre- and postinterventional DSA-series were postprocessed by PCC, and time-density curves were calculated. The parameters aneurysmal inflowvelocity, outflow-velocity and relative time-to-peak (rTTP) were calculated. Pre- and postinterventional values were compared and correlated with the occlusion rate after six months.

Results: Follow-up DSA detected 10 aneurysms occluded, meaning an occlusion rate of $91 \%$. No procedure-related morbidity and mortality was found. Flow analyses revealed a significant reduction of aneurysmal inflow- velocity and prolongation of rTTP after FDS deployment. Concerning aneurysm occlusion, the postinterventional outflow-velocity turned out to be a marginally statistically significant predictor. A definite threshold value ( -0.7 density change/s) could be determined for the outflow-velocity that allows prediction of complete aneurysm occlusion with high sensitivity and specificity $(100 \%)$.

Conclusions: Using FDS can be considered an efficient and safe therapy option in treating unruptured, intradural VADA. From in vivo flow analyses the postinterventional aneurysmal outflow-velocity turned out to be a potential predictor for later complete aneurysm occlusion. Here, it might be possible to determine a threshold value that allows prediction of aneurysm occlusion with high specificity and sensitivity. As fast, applicable and easy-to-handle tool, PCC could be used for procedural monitoring and might contribute to further treatment optimization.
\end{abstract}

\section{Keywords}

Cerebral aneurysm treatment, flow-diverting stents, vascular disease, image postprocessing

Received 15 June 2015; accepted 9 August 2015

\section{Introduction}

Dissecting aneurysms (DAs) of the intradural segment of the vertebral artery (VA) (VADAs) are a rare but important cause of subarachnoidal hemorrhage (SAH), accounting for $3 \%-7 \%$ of these cases. ${ }^{1}$ While DAs represent only $3 \%$ of all intracranial aneurysms, they account for $28 \%$ of VA aneurysms. ${ }^{2}$ Recent studies supposed the risk of bleeding from unruptured VA dissections to be higher than previously considered. ${ }^{3}$ Furthermore, VADAs have a high risk of causing ischemic events by thromboembolic vessel occlusion. ${ }^{4}$ Most intracranial VA dissections occur spontaneously in the fourth or fifth decade of life, or following minor blunt trauma. ${ }^{5}$

To sacrifice the VA by occluding the aneurysmal segment via coil embolization is a possible endovascular treatment option that is reported with high success. ${ }^{6,7}$ But this procedure harbors the risk of occluding side branches to the brainstem. Furthermore, vessel occlusion is problematic if the aneurysmal segment incorporates major branches such as the anterior inferior cerebellar artery (AICA), the posterior inferior cerebellar artery (PICA) or the anterior spinal artery.

\footnotetext{
${ }^{1}$ Department of Neuroradiology, University of Erlangen-Nuremberg, Germany

${ }^{2}$ Department of Neurosurgery, University of Erlangen-Nuremberg, Germany
} ${ }^{3}$ Department of Neurology, University of Erlangen-Nuremberg, Germany

\section{Corresponding author:}

Philipp Gölitz, Department of Neuroradiology, University of ErlangenNuremberg Schwabachanlage 6, 91054 Erlangen, Germany.

Email: philipp.goelitz@uk-erlangen.de 
As well, the deconstructive approach is limited if the dominant VA is involved without adequate collateralization from the contralateral artery, or if the contralateral artery is diseased.

Here, the development of flow-diverting stents (FDS) allows a new endovascular treatment regimen that enables the preservation of the affected vessel segment. In the literature, only a few cases are reported using FDS for treatment of unruptured VADAs, ${ }^{8,9}$ and still fewer are reported for the treatment of ruptured VADAs. ${ }^{10,11}$ Whether after FDS deployment complete aneurysm occlusion will occur, and with that the definite elimination of the risk of $\mathrm{SAH}$, is, at least at the moment, not predictable. Here, in vivo flow analyses might provide an explanatory approach by elucidating alterations of aneurysmal flow pattern, and by revealing predictive flow parameters. Recently, parametric color coding (PCC) has been introduced as a tool that allows in vivo measurements of flow dynamics in digital subtraction angiography (DSA). ${ }^{12-14}$ By this software the temporal course of density and flow changes during an entire DSA series are converted into a single, composite color-coded image. Additionally, time-density curves (TDCs) can be calculated, offering the option of obtaining quantifiable flow parameters.

The purpose of our prospective feasibility study was on the one hand to evaluate the efficiency of using FDS for the treatment of unruptured, intradural VADAs. On the other hand, by performing in vivo flow analysis using PCC, we aimed to detect specific flow alterations induced by FDS deployment that might function as a possible surrogate marker that allows us to predict later aneurysm occlusion.

\section{Methods}

After obtaining informed consent from patients, 11 consecutive patients (five women, six men) with unruptured, intradural VADAs treated by FDS were enrolled in this prospective study over a time period of 17 months. The average age was $58 \pm 10$ years (range 47-74 years). The Pipeline embolization device (PED; ev3, Plymouth, MN, USA) was used for treatment in six cases, and the SILK artery reconstruction device (SILK; BALT Extrusion, Montmorency, France) in five cases. On average, the aneurysms were $4.8( \pm 2.8) \mathrm{mm}$ high and $8.5( \pm 3.8) \mathrm{mm}$ wide. Nine aneurysms were located on the right side and two aneurysms on the left side. All aneurysms were incidental findings, except one patient who had suffered from a thromboembolic cerebellar infarction, presumably caused by the VADA. In one aneurysm the PICA originated from the aneurysm sac and in four cases the PICA originated adjacent to the aneurysm, so that the deployed FDS also covered the PICA. In three patients a dissection of the VA was known from the patient history, and follow-up imaging revealed the development of a DA. In the other cases, the diagnosis of a DA was based on the angiographic appearance with luminal dilation adjacent to a narrowed segment if additional magnetic resonance imaging (MRI) confirmed no notable circumferential vessel degeneration. Aneurysms associated with significant elongation and tortuosity of the parent artery were not included because in such cases an atherosclerotic origin can be considered.

In all patients dual anti-platelet therapy (aspirin, clopidogrel) was initiated seven days before treatment. Multi-plate testing was performed prior to therapy. After three months, clopidogrel was stopped and aspirin continued.

The day after intervention, MRI was performed to exclude ischemic events. Six months after treatment, DSA was performed in every patient to prove aneurysm occlusion. Additionally, the day prior to DSA in every patient an MRI was performed to exclude silent ischemic events that might be caused by the FDS. Moreover, directly after intervention and at the time of follow-up all patients were examined by an experienced neurologist.

\section{Image acquisition}

DSA was performed under general anesthesia on a biplane flat-detector angiographic system (Axiom Artis dBA, Siemens AG, Healthcare Sector, Forchheim, Germany). Vital parameters, i.e. heart rate and blood pressure, were monitored continuously and stabilized during intervention. Using standard angiographic methods (transfemoral route), a $6 \mathrm{~F}$ guiding catheter was positioned in the VA. Pre- and postinterventional two-dimensional (2D) DSA series were acquired at a rate of four frames per second, as is routine in our department. The initial DSA series showed in no case any vasospasm of the VA. During the interventional procedure, catheter placement, table position and frame rate remained unchanged. For image acquisition $8 \mathrm{ml}$ of contrast material (Imeron 300, Bracco Imaging, Konstanz, Germany) were injected manually by one operator at a flow rate of about $4 \mathrm{ml} / \mathrm{s}$ for 2 seconds (s).

\section{Postprocessing (PCC)}

Angiographic data were transferred to a dedicated workstation (Leonardo, Siemens AG, Healthcare Sector, Forchheim, Germany) running standard angiographic software and additional iFlow software (syngo iFlow, Siemens AG, Healthcare Sector, Forchheim, Germany). After opening the 2D DSA series in the angio mode, the color-coded images can be generated automatically. For each pixel the time between contrast material injection and maximum opacification is converted into a specific color, ranging from red to blue. Additionally, the density change over time can be displayed as a curve diagram for freely chosen points of interest (POIs). The curve shape represents the dynamic behavior of the mixture of blood and contrast material 
and illustrates the wash-in and wash-out dynamics. Based on the TDCs, the following parameters can be calculated:

- relative "time-to-peak" (rTTP) of aneurysmal filling: time from start of the curve to maximum peak measured in s, provided automatically by the software

- aneurysmal inflow: average slope of the curve within the segment before the peak, representing the inflowvelocity

- aneurysmal outflow: average slope of the curve within the segment after the peak, representing the outflow-velocity (negative values since the flow is directed out of the aneurysm)

In- and outflow are velocity indices indicating the density change over time, measured as "density change/s." Based on the electronically obtained coordinates from the curve shape, the velocity can be calculated by curve arithmetic: velocity $=\Delta y / \Delta x($ see Figure 1$)$.

\section{Image and statistical analysis}

Aneurysmal flow analysis was performed on the generated pre- and postinterventional PCC images. Prior, TDCs within the VA proximal to the aneurysm were generated and compared, confirming in this way the robustness of the manual contrast injections. Then, a POI was placed within the middle of the aneurysm sac at an identical pre- and postinterventional position and the TDCs were generated, respectively. Based on these TDCs, the aneurysmal inflow- and outflow-velocity as well as the rTTP were calculated, respectively.
Statistical analysis was performed using the software SPSS Statistics. For each pre- and postinterventional parameter the mean value and the standard deviation were calculated. The differences between the pre- and the postinterventional values were tested for significance using a two-sided Wilcoxon-test, respectively. A level of $p \leq 0.05$ was considered statistically significant, and a level of $p \leq 0.1$ as marginally significant. The pre- and postinterventional flow parameters and the aneurysm size were correlated with aneurysm occlusion using Spearman correlation, respectively. Moreover, for the postinterventional aneurysmal outflow-velocity a threshold value was determined and its sensitivity and specificity regarding complete aneurysm occlusion after six months were calculated.

\section{Results}

DSA after six months revealed 10 aneurysms completely occluded (occlusion rate 91\%); only one aneurysm showed only partial regression. No aneurysm showed progression or rupture. All PICAs covered by the FDS were delineable unchanged in the follow-up and demonstrated no occlusion. No in-stent stenosis, thrombosis or intimal hyperplasia was revealed in the follow-up. No ischemic events were observed clinically after treatment. MRI directly after intervention and MRI follow-up detected in no patients any new occurred infarctions. Complete occlusion was observed in all six aneurysms treated with the PED (occlusion rate $100 \%$ ) and in four of the five aneurysms treated with the SILK (occlusion rate $80 \%$ ).

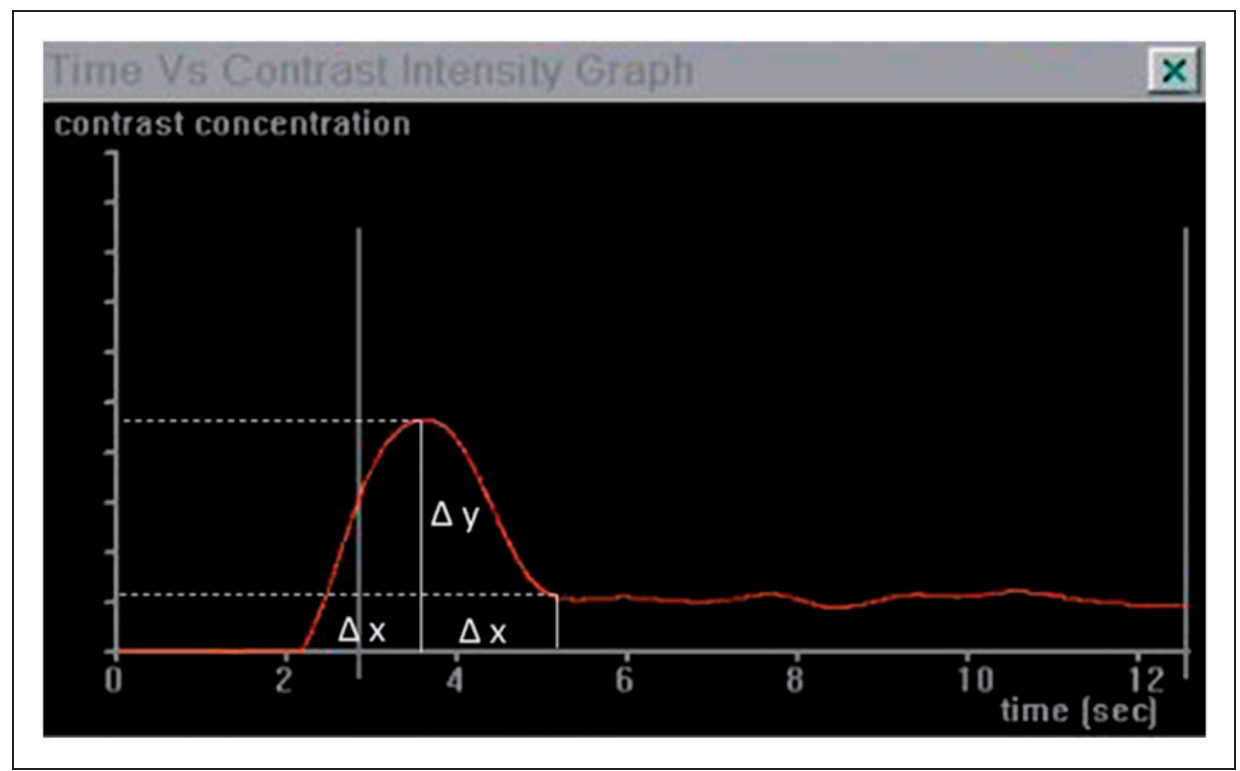

Figure 1. The initial TDC of an unruptured, intradural VADA is presented to illustrate the calculation of the flow parameters. The mean slope of the curve before the peak, representing the inflow-velocity, can be calculated by dividing the density change ( $\Delta$ y) by the time from the starting point to the peak of the curve $(\Delta x)$. Vice versa, the mean slope of the curve after the peak, representing the outflowvelocity, can be calculated by dividing the density change $(\Delta y)$ by the time from the peak to the endpoint of the curve ( $\Delta \mathrm{x})$. TDC: timedensity curve; VADA: dissecting aneurysm of the intradural segment of the vertebral artery. 


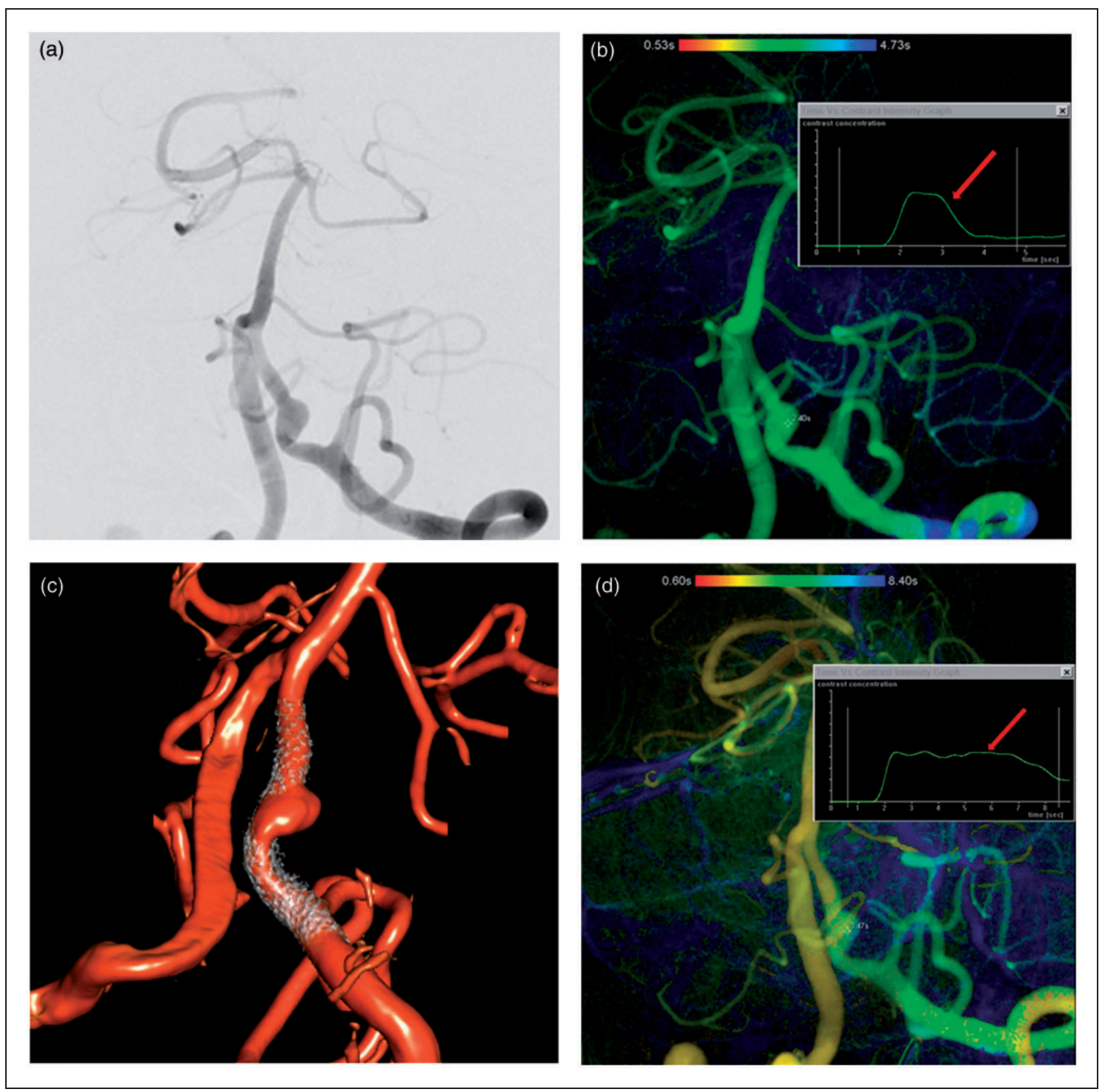

Figure 2. Two-dimensional DSA presents unruptured, intradural VADA on left VA (a). Three-dimensional DSA demonstrates correct positioning of the implanted PED (c). The initial flow analysis using PCC (b) shows a rapid aneurysmal wash-out (red arrow) with a calculated outflow-velocity of $-1 \mathrm{D} / \mathrm{s}$. After FDS deployment flow analysis (d) reveals a distinct flattening of the curve in the wash-out segment (red arrow) with a calculated outflow-velocity of $-0.2 \mathrm{D} / \mathrm{s}$. Thus, by FDS deployment a clear reduction of the aneurysmal outflowvelocity could be induced. Since the postinterventional value is less than the determined threshold value of $-0.7 \mathrm{D} / \mathrm{s}$, complete aneurysm occlusion could be expected, which was confirmed in the follow-up. DSA: digital subtraction angiography; VADA: dissecting aneurysm of the intradural segment of the vertebral artery; VA: vertebral artery; PED: Pipeline embolization device; FDS: flow-diverting stents.

PCC and calculation of the TDCs were possible in all examinations, taking about 5 minutes to generate in each case. In $91 \%$ of the postinterventional TDCs a distinct flattening of the curve in the interval after the peak was observed (see Figure 2). The mean preinterventional aneurysmal inflow-velocity was $4.9( \pm 2.5)$ $\mathrm{D} / \mathrm{s}$, the outflow-velocity $-2.2( \pm 3.6) \mathrm{D} / \mathrm{s}$ and the rTTP $1.2 \mathrm{~s}( \pm 0.6)$. After FDS deployment the mean aneurysmal inflow-velocity was reduced to $2.5( \pm 2.2)$ $\mathrm{D} / \mathrm{s}$, the outflow-velocity to $-0.5( \pm 0.6) \mathrm{D} / \mathrm{s}$ and the rTTP prolonged to $2.2 \mathrm{~s}( \pm 1.4)$. The difference between the pre- and the postinterventional values reached significance for the inflow-velocity $(p=0.005)$ and the rTTP $(p=0.013)($ Table 1$)$.
Concerning complete aneurysm occlusion, the postinterventional outflow-velocity turned out to be a marginally significant parameter $(p=0.057)$. If for the postinterventional outflow-velocity a threshold value of $-0.7 \mathrm{D} / \mathrm{s}$ was determined, aneurysm occlusion after six months was observed with a sensitivity and specificity of $100 \%$, respectively (see Figure 2).

Subgroup analysis revealed that the mean postinterventional aneurysmal outflow-velocity was -0.33 $( \pm 0.22) \mathrm{D} / \mathrm{s}$ after PED deployment and $-0.70( \pm 0.91)$ $\mathrm{D} / \mathrm{s}$ after SILK deployment. The difference between these values did not reach a level of significance in a Mann-Whitney $U$ test. 
Table 1. Aneurysmal flow parameters before and after FDS deployment.

\begin{tabular}{lllllll}
\hline & $\begin{array}{l}\text { Inflow_pre- } \\
\text { (density change/s) }\end{array}$ & $\begin{array}{l}\text { Inflow_post- } \\
\text { (density change/s) }\end{array}$ & $\begin{array}{l}\text { Outflow_pre- } \\
\text { (density change/s) }\end{array}$ & $\begin{array}{l}\text { Outflow_post- } \\
\text { (density change/s) }\end{array}$ & $\begin{array}{l}\text { rTTP_pre- } \\
\text { (s) }\end{array}$ & $\begin{array}{l}\text { rTTP_post- } \\
\text { (s) }\end{array}$ \\
\hline Mean value & 4.9 & 2.5 & -2.2 & -0.5 & 1.2 & 2.2 \\
$\begin{array}{l}\text { Standard deviation } \\
\begin{array}{l}\text { Difference Pre- vs. } \\
\text { post- value }\end{array}\end{array}$ & $p=0.5$ & 2.2 & 3.6 & 0.6 & 0.6 & 1.4 \\
\hline
\end{tabular}

FDS: flow-diverting stents; rTTP: relative time-to-peak.
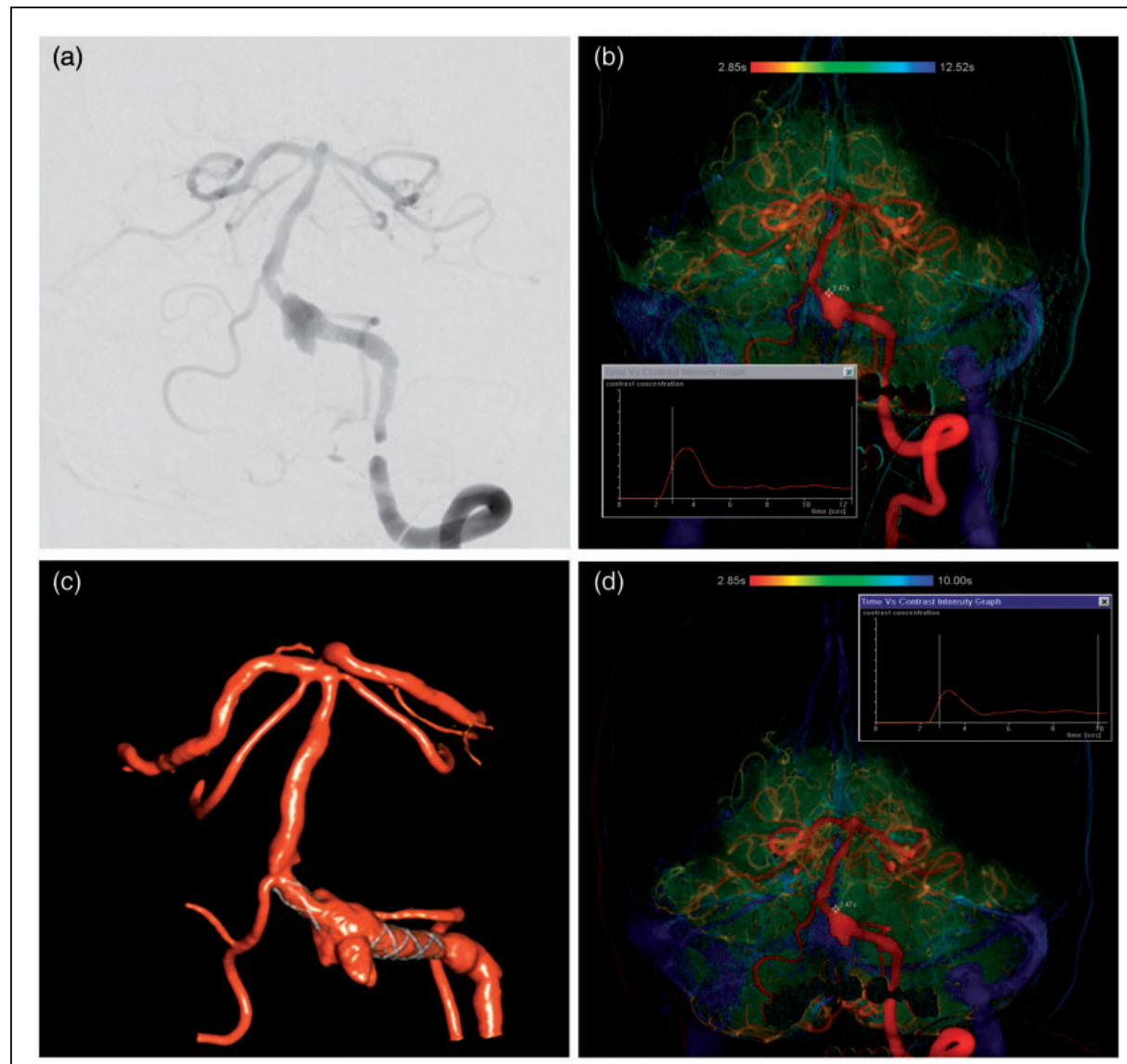

(e)

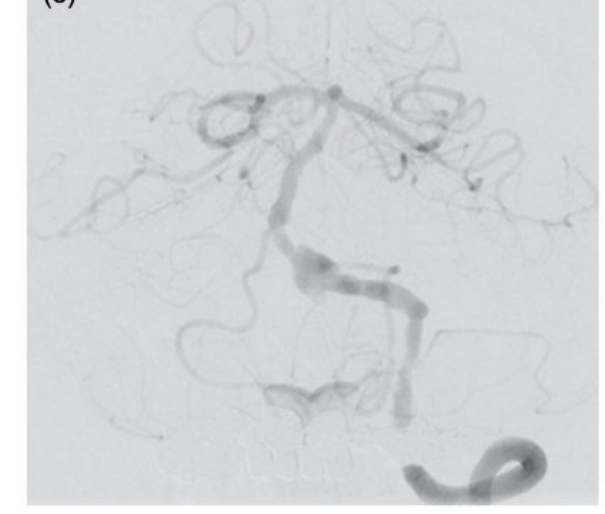

Figure 3. Two-dimensional DSA presents an unruptured, intradural VADA on left VA (a). Three-dimensional DSA after intervention confirms correct positioning of the SILK (c). Initial (b) and postinterventional (d) flow analysis reveal a nearly unchanged aneurysmal flow pattern with a constant outflow-velocity $(-1.5 \mathrm{D} / \mathrm{s})$. Since the outflow-velocity is higher than our determined threshold value $(-0.7 \mathrm{D} / \mathrm{s})$, aneurysm occlusion after six months should not be expected. Correspondingly, follow-up DSA demonstrates no aneurysm occlusion (e). DSA: digital subtraction angiography; VADA: dissecting aneurysm of the intradural segment of the vertebral artery; VA: vertebral artery. 
Table 2. List of each individual patient data.

\begin{tabular}{|c|c|c|c|c|c|c|c|c|c|c|c|c|}
\hline Patients & Age & Gender & $\begin{array}{l}\text { Aneurysm } \\
\text { Side }\end{array}$ & $\begin{array}{l}\text { Aneurysm size } \\
\text { (height } \times \text { width } \\
\text { in } \mathrm{mm} \text { ) }\end{array}$ & FDS & $\begin{array}{l}\text { Inflow } \\
\text { velocity } \\
\text { pre- } \\
\text { (density/s) }\end{array}$ & $\begin{array}{l}\text { Outflow } \\
\text { velocity pre- } \\
\text { (density/s) }\end{array}$ & $\begin{array}{l}\text { rTTP } \\
\text { pre- } \\
\text { (s) }\end{array}$ & $\begin{array}{l}\text { Inflow } \\
\text { velocity } \\
\text { post- } \\
\text { (density/s) }\end{array}$ & $\begin{array}{l}\text { Outflow } \\
\text { velocity } \\
\text { post- } \\
\text { (density/s) }\end{array}$ & $\begin{array}{l}\text { rTTP } \\
\text { post- } \\
\text { (s) }\end{array}$ & $\begin{array}{l}\text { Occlusion } \\
\text { after six } \\
\text { months } \\
\text { on DSA }\end{array}$ \\
\hline 1 & 73 & Male & Right & $9.5 \times 12.0$ & PED & 2.6 & -0.7 & 2.2 & 1.8 & -0.4 & 2.6 & Yes \\
\hline 2 & 47 & Male & Right & $4.0 \times 12.0$ & SILK & 5.1 & -0.4 & 1.1 & 0.6 & -0.3 & 4.5 & Yes \\
\hline 3 & 51 & Female & Right & $2.5 \times 4.0$ & PED & 8.0 & -4.4 & 0.6 & 7.9 & -0.7 & 0.7 & Yes \\
\hline 4 & 58 & Male & Right & $5.5 \times 10.0$ & PED & 6.8 & -0.5 & 0.8 & 4.3 & -0.1 & 1.0 & Yes \\
\hline 5 & 58 & Male & Left & $10.0 \times 13.0$ & SILK & 7.4 & -2.5 & 0.9 & 4.2 & -2.3 & 1.4 & No \\
\hline 6 & 60 & Female & Left & $3.0 \times 5.0$ & PED & 4.0 & -1.0 & 0.7 & 2.9 & -0.2 & 0.8 & Yes \\
\hline 7 & 48 & Male & Right & $6.0 \times 10.0$ & SILK & 2.3 & -0.4 & 1.6 & 1.5 & -0.3 & 3.1 & Yes \\
\hline 8 & 72 & Female & Right & $3.5 \times 5.5$ & PED & 1.4 & -0.3 & 2.1 & 0.8 & -0.2 & 4.5 & Yes \\
\hline 9 & 52 & Male & Right & $4.0 \times 12.5$ & SILK & 6.2 & -12.4 & 1.2 & 1.0 & -0.1 & 1.2 & Yes \\
\hline 10 & 50 & Female & Right & $3.0 \times 6.0$ & SILK & 1.9 & -0.6 & 1.4 & 1.3 & -0.5 & 2.2 & Yes \\
\hline 11 & 74 & Female & Right & $2.0 \times 3.0$ & PED & 7.8 & -0.9 & 0.4 & 1.6 & -0.4 & 2.3 & Yes \\
\hline
\end{tabular}

FDS: flow-diverting stents; PED: Pipeline embolization device; rTTP: relative time-to-peak; DSA: digital subtraction angiography.

In the one case of incomplete aneurysm occlusion the outflow-velocity was almost unchanged $(-1.5 \mathrm{D} / \mathrm{s})$ despite FDS implantation (see Figure 3). A special attribute in that case that has to be considered is that the PICA originated from aneurysm sac.

Individual patient data are summarized in Table 2.

\section{Discussion}

In our study the treatment of unruptured, intradural VADAs by FDS deployment turned out to be a safe and effective therapy option that makes sacrificing the parent artery unnecessary. We found no morbidity or mortality associated with FDS implantation. Up to now, there have been only a few series with small sample sizes using FDS for the treatment of unruptured VADAs. In line with our results, no thromboembolic complications or stent thrombosis are reported after FDS treatment. ${ }^{9}$ A recently published meta-analysis found for the endovascular reconstructive treatment of unruptured, vertebrobasilar DAs a perioperative morbidity of $7 \%$, a perioperative mortality of $5 \%$ and a long-term good clinical outcome rate of $94 \% .{ }^{15}$ The higher perioperative morbidity and mortality in comparison with our results might be explained by the fact that the reconstructive treatment techniques were not restricted to FDS implantations, but also stent-assisted coil embolizations were subsumed, which might be associated with a higher perioperative risk. The aneurysm occlusion rate in our study was $91 \%$ after six months. For comparison, the aneurysmal occlusion rate of VADAs as reported in the literature varies from $60 \%{ }^{8}$ to $100 \%,{ }^{9}$ based on the experience from five ${ }^{8}$ or four cases. ${ }^{9}$ The above-mentioned metaanalysis found angiographically a long-term occlusion rate of $68 \%$ after reconstructive treatment of unruptured, vertebrobasilar DAs. ${ }^{15}$ Our higher occlusion rate might be explained by the fact that if stent-assisted coil embolizations as reconstructive techniques are included, aneurysm recanalization can occur because of coil compaction. The observation of a male predominance and predominant right-sided localization of VADAs in our study is in line with the literature. ${ }^{5,16}$

To further elucidate the phenomenon of a high aneurysmal occlusion rate in our series, we performed in vivo flow analysis using the recently introduced method of PCC, which is suitable for the postprocessing of DSA series. This commercially available software (syngo iFlow; Siemens Healthcare, Forchheim, Germany) allows in vivo flow analysis without the need for additional x-ray or contrast medium application, since regular DSA series are used. This software converts the time between contrast material injection and maximum opacification into a specific color, ranging from red to blue. Additionally, in freely chosen POIs TDCs can be generated. From curve data flow parameters like rTTP and flow velocity can be calculated by curve arithmetic (see Figure 1). In consequence, flow alterations induced by FDS implantation can be visualized in a noninvasive way and quantified by TDC analysis. The feasibility of using PCC for aneurysmal flow analysis has been demonstrated in an animal study, ${ }^{17}$ and in humans the potential of PCC for predicting aneurysm occlusion after FDS treatment has been reported for saccular aneurysms. ${ }^{18}$ But so far, there are, to the best of our knowledge, no studies using PCC for the evaluation of unruptured, intradural VADAs.

Our flow analyses revealed a significant reduction of aneurysmal inflow- velocity and a significant prolongation of aneurysmal rTTP induced by FDS deployment. Concerning the aneurysmal occlusion rate after six months, the postinterventional outflow-velocity turned out to be a marginally statistically significant parameter. There was no significant difference between SILK and PED concerning the reduction of the aneurysmal 
outflow-velocity. In our series it was possible to determine a definite threshold value $(-0.7 \mathrm{D} / \mathrm{s})$ for the postinterventional outflow-velocity, which allows prediction of aneurysm occlusion with a sensitivity and specificity of $100 \%$, respectively. Based on that, unruptured, intradural VADAs seem suitable for FDS treatment insofar as by FDS implantation a substantial reduction of the aneurysmal outflow-velocity was achievable in nearly all cases. In one case, no aneurysm occlusion after six months was detected, the flow analysis revealed an almost unchanged aneurysmal outflow-velocity despite FDS implantation. However, in that case, the PICA originated from the aneurysm dome, which is an important confounding factor since the chance of obliteration can be considered low if a vessel emanates from the aneurysm. In such cases, the deployment of a second device could be considered for further treatment. From that point, PCC as a fast, applicable and easy-to-handle tool could be used for procedural monitoring, especially since it can be displayed directly within the angio suite. In cases where the aneurysmal outflow-velocity is not reduced substantially or our identified threshold value is not reached by FDS deployment, the implantation of a second device could be considered. If a major branch, i.e. the PICA, emanates from the aneurysm sac, it seems necessary to implant a second device to obtain sufficient outflow reduction, but here our results are limited by the small sample size. On the other hand, by performing in vivo flow analysis the unnecessary implantation of a second device could be avoided, which in turn could contribute to patient safety.

As an advantage in contrast to computational fluid dynamics (CFD) simulations, PCC is less time consuming, can be performed directly within the angio suite and is not limited by simplifications of hemodynamic features for technical reasons or lack of patient-specific information on physiological flow velocity. ${ }^{19}$ Also subtle changes in stent porosity caused by deformity in curved cerebral vessels cannot be incorporated into CFD simulations. Moreover, CFD simulations harbor general limitations like the assumption of blood as a Newtonian fluid.

Certainly, an important limitation of our study is that in the one case where the aneurysm was not occluded, the PICA originated from the aneurysm dome, which is an important confounding factor making later aneurysm obliteration difficult. Furthermore, our results are limited by the small sample size, but unruptured VADAs are rare findings. Additional cases and flow analyses should be added to our series to further substantiate our results. Moreover, long-term results have to be awaited. An object for further investigations could be the use of FDS combined with in vivo flow analysis in case of acute ruptured VADAs.

\section{Conclusions}

Based on our data, the implantation of a FDS can be considered an efficient and safe therapy option in treating unruptured, intradural VADAs that makes sacrificing the parent artery unnecessary. Performing in vivo flow analyses using PCC offers the option of gaining noninvasively flow parameters, i.e. the postinterventional aneurysmal outflow-velocity, which could function as predictors for later complete aneurysm occlusion.

\section{Disclosure of conflicting interests}

The authors declared no potential conflicts of interest with respect to the research, authorship, and/or publication of this article.

\section{Funding}

The author(s) received no financial support for the research, authorship, and/or publication of this article.

\section{References}

1. Sasaki O, Ogawa H, Kioke T, et al. A clinicopathologic study of dissecting aneurysms of the intracranial vertebral artery. J Neurosurg 1991; 75: 874-882.

2. Luo CB, Chang CY, Teng MM, et al. Endovascular treatment of ruptured vertebral dissecting aneurysms with electrodetachable coils. J Chin Med Assoc 2005; 68: $578-584$.

3. Naito I, Iwai T and Sasaki T. Management of intracranial vertebral artery dissections initially presenting without subarachnoid haemorrhage. Neurosurgery 2002; 51: 930-937.

4. Flemming KD, Wiebers DO, Brown Jr, RD, et al. The natural history of radiographically defined vertebrobasilar nonsaccular intracranial aneurysms. Cerebrovasc Dis 2005; 20: 270-279.

5. Yamaura A, Watanabe Y and Saeki N. Dissecting aneurysms of the intracranial vertebral artery. $J$ Neurosurg 1990; 72: 183-188.

6. Peluso JP, van Rooij WJ, Sluzewski M, et al. Endovascular treatment of symptomatic intradural vertebral dissecting aneurysms. AJNR Am J Neuroradiol 2008; 29: 102-106.

7. Kashiwazaki D, Ushikoshi S, Asano T, et al. Long-term clinical and radiological results of endovascular internal trapping in vertebral artery dissection. Neuroradiology 2013; 55: 201-206.

8. de Barros Faria M, Castro RN, Lundquist J, et al. The role of the pipeline embolization device for the treatment of dissecting intracranial aneurysms. AJNR Am $J$ Neuroradiol 2011; 32: 2192-2195.

9. Yeung TW, Lai V, Lau HY, et al. Long-term outcome of endovascular reconstruction with the Pipeline embolization device in the management of unruptured dissecting aneurysms of the intracranial vertebral artery. J Neurosurg 2012; 116: 882-887.

10. Ducruet AF, Crowley RW, Albuquerque FC, et al. Reconstructive endovascular treatment of a ruptured vertebral artery dissecting aneurysm using the Pipeline embolization device. J Neurointerv Surg 2013; 5: e20.

11. Narata AP, Yilmaz H, Schaller K, et al. Flow-diverting stent for ruptured intracranial dissecting aneurysm of vertebral artery. Neurosurgery 2012; 70: 982-988. discussion 988-989. 
12. Strother CM, Bender F, Deuerling-Zheng $\mathrm{Y}$, et al. Parametric color coding of digital subtraction angiography. AJNR Am J Neuroradiol 2010; 31: 919-924.

13. Struffert T, Deuerling-Zheng Y, Engelhorn T, et al. Monitoring of balloon test occlusion of the internal carotid artery by parametric color coding and perfusion imaging within the angio suite: First results. Clin Neuroradiol 2013; 23: 285-292.

14. Gölitz P, Struffert T, Lücking H, et al. Parametric color coding of digital subtraction angiography in the evaluation of carotid cavernous fistulas. Clin Neuroradiol 2013; 23: 113-120.

15. Sönmez Ö, Brinjikji W, Murad MH, et al. Deconstructive and reconstructive techniques in treatment of vertebrobasilar dissecting aneurysms: A systematic review and metaanalysis. AJNR Am J Neuroradiol 2015; 36: 1293-1298.

16. Dabus G, Lin E and Linfante I. Endovascular treatment of fusiform intracranial vertebral artery aneurysms using reconstructive techniques. J Neurointerv Surg 2014; 6: 589-594.

17. Struffert T, Ott S, Kowarschik M, et al. Measurement of quantifiable parameters by time-density curves in the elastase-induced aneurysm model: First results in the comparison of a flow diverter and a conventional aneurysm stent. Eur Radiol 2013; 23: 521-527.

18. Gölitz P, Struffert T, Rösch J, et al. Cerebral aneurysm treatment using flow-diverting stents: In-vivo visualization of flow alterations by parametric colour coding to predict aneurysmal occlusion: Preliminary results. Eur Radiol 2015; 25: 428-435.

19. Kono K, Shintani A, Fujimoto T, et al. Stent-assisted coil embolization and computational fluid dynamics simulations of bilateral vertebral artery dissecting aneurysms presenting with subarachnoid hemorrhage: Case report. Neurosurgery 2012; 71: E1192-E1200. discussion E1200-E1201. 